\title{
Performance Analysis with Wireless Power Transfer Constraint Policies in Full-Duplex Relaying Networks
}

\author{
Hoang-Sy Nguyen ${ }^{1,2}$, Miroslav Voznak ${ }^{1,2}$, Minh-Tung Nguyen ${ }^{3}$, Lukas Sevcik ${ }^{2}$ \\ ${ }^{1}$ Faculty of Electrical and Electronics Engineering, Ton Duc Thang University, \\ 19 Nguyen Huu Tho St., Tan Phong Ward, Dist. 7, Ho Chi Minh City, Vietnam \\ ${ }^{2}$ Faculty of Electrical Engineering and Computer Science, Technical University of Ostrava, \\ 17. listopadu 2172/15, 70833 Ostrava-Poruba, Czech Republic \\ ${ }^{3}$ Binh Duong University, \\ Binh Duong Province, Vietnam \\ voznak@ieee.org
}

\begin{abstract}
In practice, full-duplex (FD) transmission mode not only helps extend the coverage but also lengthen network lifetime. In this paper, we develop wireless power supply policies, namely separated power (SP) and harvested power (HP) to propose a flexible architecture at the relay node in FD decode-and-forward (DF) relaying networks considering time switching-based relaying protocol (TSR) to achieve optimal time used for a communication process. This transmission mode requires more processing procedure at the relay, i.e. antenna installations and radio frequency (RF) self-interference cancellation. We evaluate the optimal power constraints in case of SP and HP to achieve better power consumption efficiency at the relay node. More importantly, closed-form expressions for outage probability and throughput are provided, and we also use numerical and simulation results to compare SP with HP.
\end{abstract}

Index Terms-Full-duplex; energy harvesting; selfinterference; wireless power allocation; time switching-based relay.

\section{INTRODUCTION}

In wireless communications, full-duplex (FD) transmission mode, which allows the transmitter and receiver to be operated simultaneously in the same frequency band, has attracted much research interest in recent years [1].

In principle, thanks to the use of FD, the spectral efficiency in advanced communication systems can be significantly increased compared to half-duplex (HD) mode. Besides that, FD mode is considered as an attractive mechanism to enhance data rate to satisfy the requirements of high transmission rate. Due to the ability to transfer both information and energy via RF signals at the same time, energy harvesting (EH) plays an important role in simultaneous wireless information and power transfer in the same circuit [2]-[4]. A principal application scenario for simultaneously wireless information and power transfer (SWIPT) so-called wireless-powered relaying (WPR) was

Manuscript received 16 October, 2016; accepted 21 May, 2017.

The research received a financial support from the SGS grant No. SP2017/174, VSB-Technical University of Ostrava, Czech Republic. put forward, in which a relay powered via RF assists the communication between the destination and the source node. In several previous studies on WPR, they mainly focused on HD relaying and the implementation of two mechanisms, namely time switching-based relaying (TSR) and power splitting-based relaying (PSR) [5]-[7]. The time switchingbased FD wireless-powered transmission was investigated, in [8], [9].

Furthermore, FD mode has more benefits in comparison with HD mode in terms of self-interference. There was a rise in noise floor because of the interfering signal according to the model of self-inference in [10]. In such a model, better self-interference cancellation and self-interference channel estimation are achieved thanks to higher transmit power.

In addition, there are other works on interference cancellation methods which were considered in [11]-[14]. In [11], the authors put forward a scheme in terms of interference aware routing for vehicular networks [12], and they continued proposing a robust scheme for interference mitigation during mobile transmissions in vehicular networks in [13]. Furthermore, power control was evaluated to reduce harmful secondary interference affecting primary users in cognitive networks. In [14], the work focused on combining interference cancellation techniques with access control to reduce the chance of packet loss and packet delay.

However, power allocation was not addressed comprehensively in the aforementioned studies in terms of opportunistic EH-assisted relay schemes. Motivated from these limitations, in this paper, we focus on power supply policies in DF FD relaying networks, where we try to achieve optimal time EH. Therefore, our main contributions can be summarized as follows:

- Closed-form expressions for outage probability and the delay-tolerant and delay-limited throughput over independent and identically distributed Rayleigh fading channels are achieved;

- The simulation results are given in terms of some parameters like self-interference, power allocation 
schemes, transmit power at source node and time switching schemes for the EH protocol and FD mode. Additionally, we also compare separated power (SP) with harvested power (HP) mode;

- The proposed EH architecture helps boost the throughput performance. Furthermore, as we derive the optimal time, the optimal transmission rate is derived for HP mode.

The paper is organized as follows. The system model is illustrated in Section II. In Section III, a relaying network is investigated with the optimal power constraints for the relay. We provide the simulation results in Section IV. Finally, Section V draws a conclusion for the paper.

Notation: $\gamma$ denotes signal-to-noise-ratio (SNR) of specific link. $F_{X}($.$) and f_{X}($.$) represent the cumulative$ distribution function (CDF) and probability distribution function $(\mathrm{PDF})$ of the variable $X$, respectively. $\operatorname{Pr}($.$) is the$ outage probability function. $E($.$) illustrates expectation$ operation and $K_{1}($.$) is the first order modified Bessel$ function.

\section{SYSTEM MODEL}

In Fig. 1, a two-hop system is considered, in which a power constrained relay (R) assists the communication between a source $(\mathrm{S})$ and a destination node $(\mathrm{D})$ due to the impossible communication between (S) and (D). It is assumed that one antenna is equipped at (S) and (D) node while $(\mathrm{R})$ consists of one receiving and one transmitting antenna. The channel coefficients of three different links, including S-R (SR), R-D (RD) and the residual loopinterference (LI) are denoted as $h, g$ and $f$, respectively. The channel power gains $|h|^{2},|g|^{2},|f|^{2}$ are exponentially distributed random variables (RVs) with means $\Omega_{h}=d_{S R}^{-m}$, $\Omega_{g}=d_{R D}^{-m}$, and $\Omega_{f}=d_{L I}^{-m}$. Let us denote the distances between ( $\mathrm{S})$ and (R), (R) and (D) as $d_{S R}, d_{R D}$, respectively. Meanwhile, $d_{L I}$ is denoted as the distance between two antennas at (R), and $m$ is the path-loss exponent. It is assumed that all nodes are affected by the additive white Gaussian noise (AWGN) which is $N_{0}$ with variance $\sigma^{2}$. Furthermore, the quasi-static channel model with ideal channel state information (CSI) is also assumed at (S) and (R). In principle, CSI could be achieved by various techniques, e.g., the pilot assisted channel reverse channel training. Note that receiving and transmitting information at (R) are carried out at the same frequency band, which leads to residual self-interference, $|f|^{2}$ regardless of the deployment of self-interference cancellation at (R).

In this paper, TSR protocol for EH is considered, in which $T$ denotes the time block for an information transfer period. During the first phase, transmitting energy accounts for $\alpha T$, in which the $\mathrm{EH}$ receiver exploits the RF signal and stores the direct current to the capacitor, while in the second phase, the residual amount of time, $(1-\alpha) T$ is used for transmitting information. Simultaneously, the received signal is decoded before transmitting antenna forwards the decoded signal to (D). Each variable corresponds with the SNR, in which single-hop transmission is carried out over the distinct channel to optimize energy efficiency.

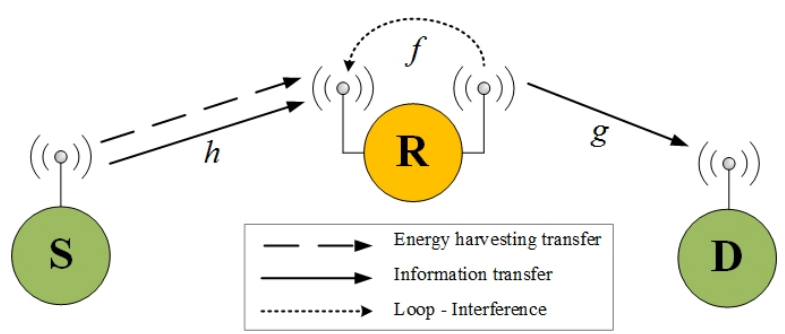

Fig. 1. The system model for the energy harvesting FD relaying networks.

We denote $P_{S}$ and $P_{R}$ as the transmit power at (S) and (R), respectively. Besides, they are considered as individual constraints, i.e., $P_{S} \leq 1$ and $P_{R} \leq 1$, respectively. In conventional networks, the received signal at the relay can be computed as

$$
y_{R}=h x_{S}+f x_{R}+N_{0},
$$

where energy symbols of both source and relay are denoted by $E\left\{\left|x_{S}\right|^{2}\right\}=P_{S}, \quad E\left\{\left|x_{R}\right|^{2}\right\}=P_{R}, \quad$ respectively. Each variable corresponds with the SNR, in which single-hop transmission is carried out over the distinct channel to optimize energy efficiency.

Regarding the cellular down-link transmission, the high SNR at relay node in case of small noise term can be computed as [15]

$$
\gamma_{R}=\frac{P_{S}|h|^{2}}{P_{R}|f|^{2}+\sigma^{2}}=\frac{P_{S}|h|^{2}}{P_{R}|f|^{2}} .
$$

Likewise, the received SNR at the destination node can be written as

$$
\gamma_{D}=P_{R}|g|^{2} / \sigma^{2}
$$

After deploying DF relay-assisted protocol, the overall end-to-end SNR can be expressed by

$$
\gamma_{e q}=\min \left\{\gamma_{R}, \gamma_{D}\right\}
$$

\section{WIRELESS POWER SUPPly POLICIES FOR RELAY NODE}

In practice, optimal power constraints at $(\mathrm{S})$ and $(\mathrm{R})$ are separated. Conventionally, we set the transmit power at (S) and $(\mathrm{R})$ as $\left\{P_{S}=1, P_{R}=1\right\}$. Therefore, the instantaneous rate of FD relaying in DF protocol can be calculated as

$$
R_{1}=B \beta \log _{2}\left(1+\gamma_{e q}\right)
$$

where $\beta=1 / 2$ and $\beta=(1-\alpha)$ are for SP and HP mode, respectively and $B$ is the signal bandwidth. 
In terms of the outage probability performance analysis, $P_{\text {out }}$, if the given target rate is higher than the system data rate. Consequently, the outage probability corresponds with the given target rate $R_{0}(\mathrm{bps} / \mathrm{Hz})$ which can be given by

$$
P_{\text {out }}^{k}\left(R_{1}<R_{0}\right) \underset{k \in\{S P, H P\}}{=} \operatorname{Pr}\left(\gamma_{\text {eq }}^{k}<\gamma_{0}\right),
$$

where we determine two cases, including SP and HP. This condition is modelled as the SNR threshold, $\gamma_{0}=2^{R_{0}}-1$.

\section{A. Separated Power (SP) Mode}

By using optimal power constraints, we can optimize the instantaneous rate. Due to the high transmission rate, the optimal power at $(\mathrm{S}), P_{S}^{*}$ and at $(\mathrm{R}), P_{R}^{*}$ can be derived by solving the optimization below

$$
\left(P_{S}^{*}, P_{R}^{*}\right)=\arg \max \left(R_{1}\right)
$$

where $0 \leq\left(P_{S}, P_{R}\right) \leq 1$. It is assumed that CSI is known at (R) and each SNR value stems from the average received power at (R). Therefore, the above expression can be rewritten as:

$$
\left\{\begin{array}{l}
\frac{P_{S}^{*}|h|^{2}}{P_{R}^{*}|f|^{2}}=P_{R}^{*} \frac{|g|^{2}}{\sigma^{2}}, \quad 0 \leq\left(P_{S}^{*}, P_{R}^{*}\right) \leq 1 . \\
P_{S}^{*}=1,
\end{array}\right.
$$

The proposed optimization problem can be solved as

$$
P_{R}^{*}=\min \left\{P_{S}^{*}, \sqrt{\frac{\sigma^{2}|h|^{2}}{|g|^{2}|f|^{2}}}\right\} .
$$

Likewise, we obtain the average optimal transmit power at relay as

$$
\bar{P}_{R}^{*}=\min \left\{\bar{P}_{S}^{*}, \sqrt{\frac{\sigma^{2} \Omega_{h}}{\Omega_{g} \Omega_{f}}}\right\}
$$

Proposition 1: For system performance analysis, the outage probability can be analytically calculated by

$$
P_{\text {out }}^{S P}\left(\gamma_{0}\right)=1-\frac{P_{S} \Omega_{h}}{P_{S} \Omega_{h}+P_{R} \Omega_{f} \gamma_{0}} \exp \left(-\frac{\sigma^{2} \gamma_{0}}{P_{R} \Omega_{g}}\right) .
$$

\section{Proof: See in Appendix A.}

\section{B. Harvested Power (HP) Assisted Relay}

Let us consider the second phase, where the received signal is forwarded to (D) after being decoded by (R). In such scenario, $(R)$ is capable of scavenging energy from the received RF signal due to not having any fixed power supplies (i.e. batteries). Thus the received signal at (R) can be computed as [6]

$$
P_{R}=\frac{E_{h}}{(1-\alpha) T}=\rho P_{S}|h|^{2},
$$

where the energy conversion efficiency is denoted by $0<\eta<1$. In addition, this coefficient illustrates the impact of EH circuitry, the harvested energy is denoted by $E_{h}=\eta \alpha P_{S}|h|^{2} T$, and $\rho=\eta \alpha(1-\alpha)^{-1}$

Based on (2), the high SNR at relay can be rewritten as

$$
\gamma_{R}=\frac{P_{S}|h|^{2}}{\rho P_{S}|h|^{2}|f|^{2}} .
$$

Next, the end-to-end SNR can be computed as [21]

$$
\gamma_{e q}^{H P}=\min \left\{\gamma_{R}, \gamma_{D}\right\}=\min \left\{\frac{1}{\rho|f|^{2}}, \rho \frac{P_{S}}{\sigma^{2}}|h|^{2}|g|^{2}\right\} .
$$

To achieve optimal transmission rate, we have

$$
\frac{1}{\eta \alpha(1-\alpha)|f|^{2}}=\eta \alpha(1-\alpha) \frac{P_{S}}{\sigma^{2}}|h|^{2}|g|^{2} \text {. }
$$

After some simple manipulations, the optimal time switching coefficient can be determined as

$$
\alpha_{o p t}=\left[\frac{2 \eta \frac{P_{S}}{\sigma^{2}} \Omega_{h} \Omega_{f}}{\left(\sqrt{1+4 \frac{P_{S}}{\sigma^{2}} \frac{\Omega_{h} \Omega_{f}}{\Omega_{g}}}-1\right)}+1\right]^{-1} .
$$

Remark 1: By choosing the value of $\alpha_{\text {opt }}$ and its effect on the instantaneous rate of the network, the (S) and (R) can harvest more energy but reduce the throughput at (D).

Proposition 2: The outage probability in EH-assisted networks can be calculated as

$$
P_{\text {out }}^{H P}\left(\gamma_{0}\right)=1-L \times \Psi K_{1}(\Psi)
$$

where $\Psi=2 \sigma^{2} \sqrt{\gamma_{0}\left(\rho P_{S} \Omega_{h} \Omega_{g}\right)^{-1}}, L=1-\exp \left(-\frac{1}{\rho \gamma_{0} \Omega_{f}}\right)$.

Proof: See in Appendix B.

\section{Asymptotic Outage Probability Analysis}

So as to simplify the approximation, upper bounds in case of SP and HP for outage probability are derived. First let us start with SP case.

\section{1) SP Case}

In order to provide some practical insights into the fundamental impact of self-interference, we now expand the low interference system. In an ideal scenario of FD systems, we assume that without significant loss of generality, $\left(\Omega_{f} \rightarrow 0\right)$. Therefore, the outage probability can be upper bounded as 


$$
P_{\text {out }}^{S P}{ }^{U p p e r}\left(\gamma_{0}\right) \approx 1-\exp \left(-\frac{\sigma^{2} \gamma_{0}}{P_{R} \Omega_{g}}\right) .
$$

\section{2) HP Case}

In the high-power regime, $\left(P_{S} \rightarrow \infty\right)$, we apply the approximation of $\Psi K_{1}(\Psi) \approx \Psi \frac{1}{\Psi}=1 \quad$ according to $(\Psi \rightarrow 0)$. Thus, we can upper bound the outage probability as follows

$$
P_{\text {out }}^{H P}{ }^{U p p e r}\left(\gamma_{0}\right) \approx \exp \left(-\frac{1}{\rho \gamma_{0} \Omega_{f}}\right) .
$$

\section{Throughput Performance}

\section{1) Delay-Limited Transmission}

We first consider the delay-limited throughput, we have to evaluate the outage probability. As a result, at the fixed rate, $R_{0}$, we have

$$
\tau_{L M}^{k} \underset{k \in\{S P, H P\}}{=} \beta\left(1-P_{o u t}^{k}\right) R_{0} .
$$

\section{2) Delay-Tolerant Transmission}

Regarding the delay-tolerant throughput, we calculate the ergodic capacity, $C^{k}, k \in\{S P, H P\}$ at (D). Note that (S) requires less transmission rate than or equal to the derived ergodic capacity. Thus, it does not require CSI at either (S) or (R) to achieve ergodic capacity. The obtained fixed transmission rate at (S) corresponds with the ergodic capacity. Therefore, the throughput, $\tau_{T O}^{k}$, at (D) can be written as

$$
\tau_{T O}^{k} \underset{k \in\{S P, H P\}}{=} \beta C^{k}
$$

where $C^{k}=E_{|h|^{2},|g|^{2},|f|^{2}} \log _{2}\left(1+\gamma_{e q}^{k}\right)$ for both case in (4). It is noted that ergodic capacity at (D) can be computed as

$$
C_{k \in\{S P, H P\}}^{k} \frac{1}{\ln 2} \int_{x=0}^{\infty} \frac{1-P_{\text {out }}^{k}(x)}{1+x} d x .
$$

\section{Simulation Results}

Some numerical results in these experiments are presented to confirm the analytical expressions derived in the above sections and the optimal throughput for the optimal power constraints with different parameters. To analyse the outage performance, the source transmission rate is set to fixed rate, e.g., $R_{0}=2(\mathrm{bps} / \mathrm{Hz})$ and hence the SNR threshold causing outage is given by $2^{R_{0}}-1=3$. The transmit power at $(\mathrm{S})$ is $P_{S}=1$. The EH efficiency is set $\eta=0.5$. Besides, a normalized system bandwidth, is set $B=1$.

For simplicity, similar noise variances are assumed at all nodes, i.e., $\sigma^{2}=0.5$. In these simulations, we denote $\bar{\gamma}_{S R}=\Omega_{h}, \bar{\gamma}_{R D}=\Omega_{g}$ and $\bar{\gamma}_{L I}=\Omega_{f}$.
In Fig. 2 and Fig. 3, the analytical results for throughput are depicted under different SNRs of the (S)-(R) hop with $\bar{\gamma}_{S R}=\bar{\gamma}_{R D}$. In case of SP, we consider $\alpha=0.5$ while with HP mode, optimal time switching-based $\alpha_{o p t}$ is chosen for performance evaluation.

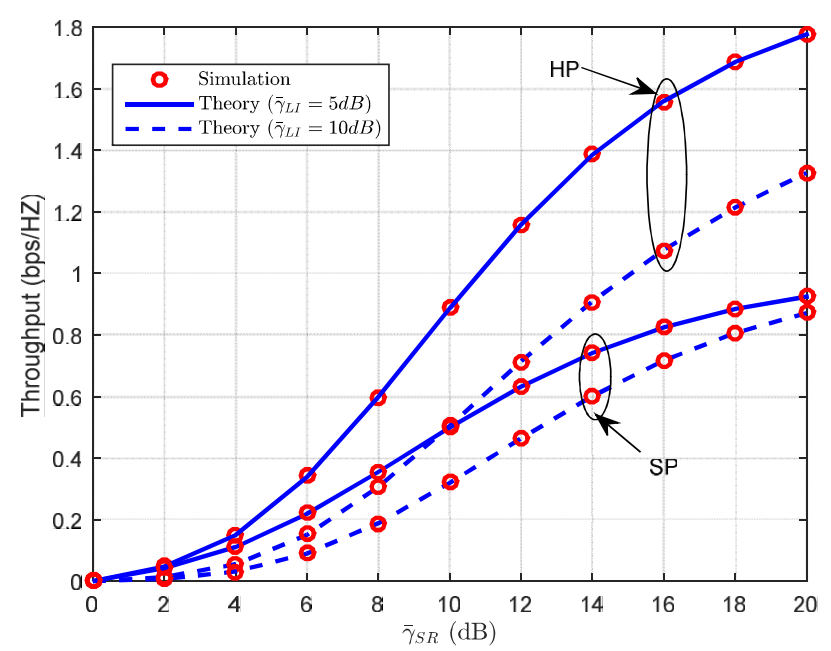

Fig. 2. Theory and simulation results of throughput vs. $\bar{\gamma}_{S R}$ under different impacts of self-interference $\bar{\gamma}_{L I}=5 \mathrm{~dB}$ or $\bar{\gamma}_{L I}=10 \mathrm{~dB}$ and $\alpha_{o p t}, \bar{\gamma}_{S R}=\bar{\gamma}_{R D}$ (Delay-limited mode).

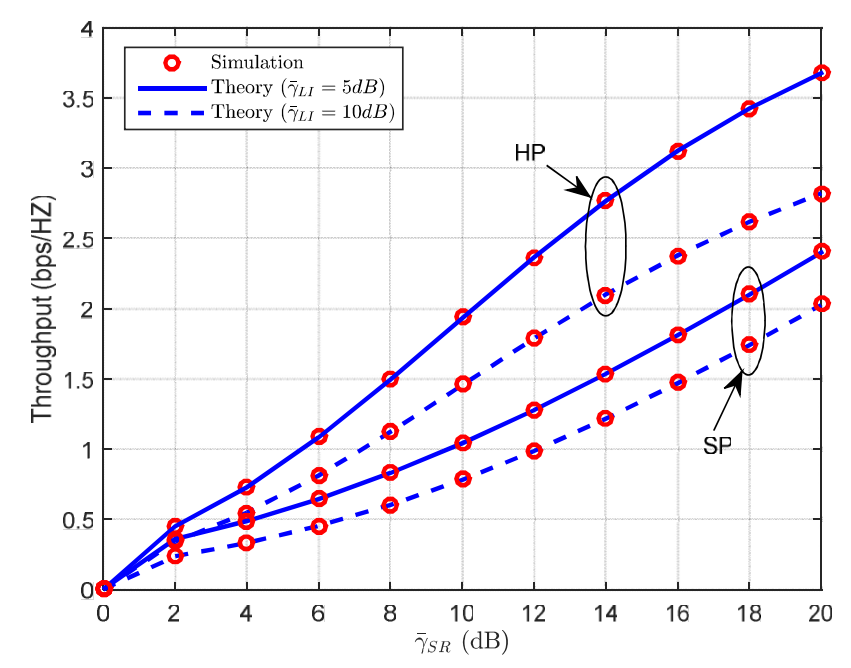

Fig. 3. Theory and simulation results of throughput vs. $\bar{\gamma}_{S R}$ under different impacts of self-interference $\bar{\gamma}_{L I}=5 \mathrm{~dB}$ or $\bar{\gamma}_{L I}=10 \mathrm{~dB}$ and $\alpha_{o p t}, \bar{\gamma}_{S R}=\bar{\gamma}_{R D}$ (Delay-tolerant mode).

Considering the impact of time switching coefficients, Fig. 4 and Fig. 5 illustrate the optimal throughput obtained with the optimal time factor of HP and also the delay-limited throughput and delay-tolerant throughput, respectively under different time allocation coefficients.

In the considered protocol, there is an increase in throughput when $\alpha$ climbs from 0 to the optimal value, $\alpha=0.1$, contributing to best performance compared to SP $\alpha=0.5$. However, it begins falling, as $\alpha$ rises from the optimal value. As the values of $\alpha$ are smaller than the optimal time, $\alpha_{\text {opt }}$, less time is utilized for $\mathrm{EH}$.

These figures confirm that self-interference factors have an important impact on throughout at every value of time 
factors. Interestingly, in both transmission modes, a reasonable choice of $\alpha$ for HP is to enable the considered protocol to satisfy a better throughput performance in many cases of self-interference.

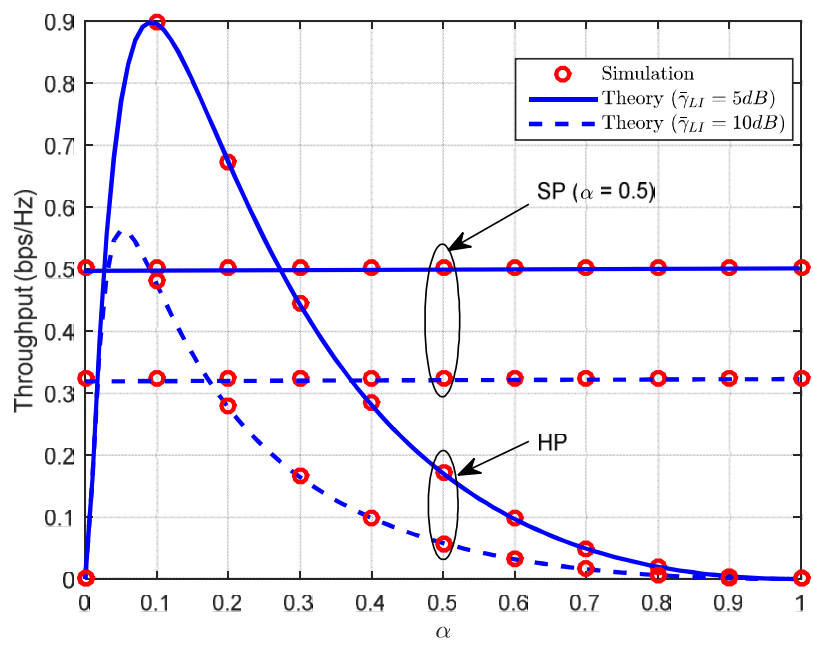

Fig. 4. Throughput performance versus time switching coefficients with $\bar{\gamma}_{S R}=\bar{\gamma}_{R D}=10 \mathrm{~dB}$ in the delay-limited mode.

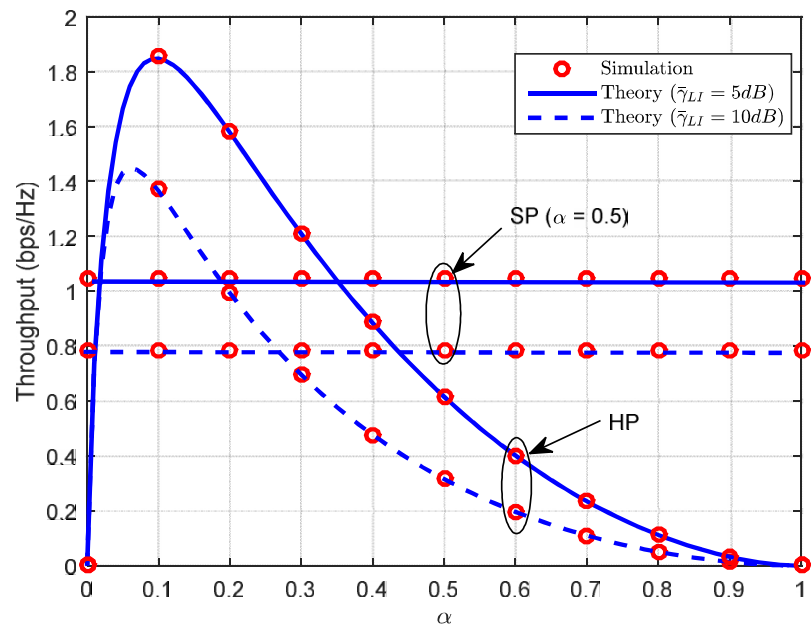

Fig. 5. Throughput performance versus time switching coefficients with $\bar{\gamma}_{S R}=\bar{\gamma}_{R D}=10 \mathrm{~dB}$ in the delay-tolerant mode.

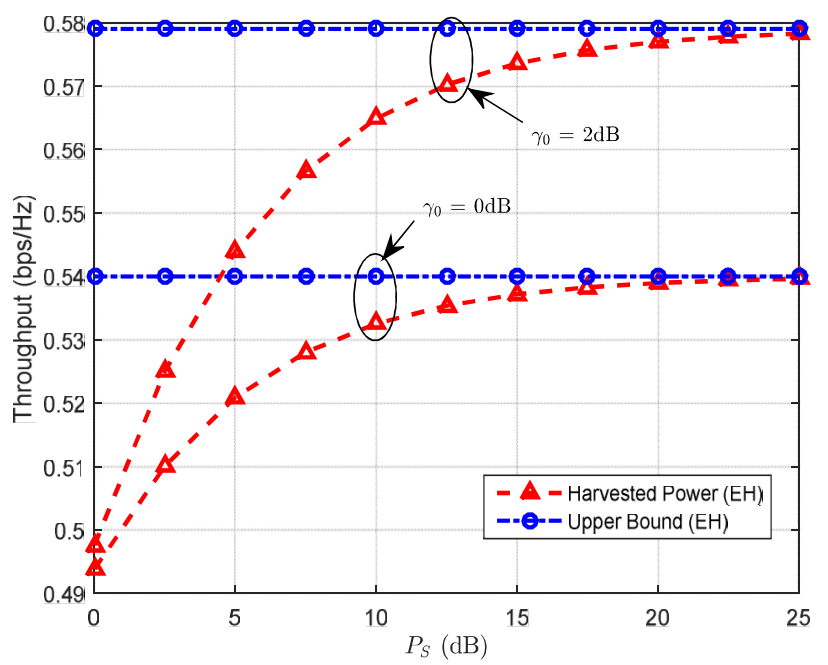

Fig. 6. The asymptotic throughput performance with performance with different transmit power in the delay-limited mode and $\bar{\gamma}_{S R}=\bar{\gamma}_{R D}=10 \mathrm{~dB}, \bar{\gamma}_{L I}=5 \mathrm{~dB}$.
Furthermore, the performance of HP reaches the peak and then declines slightly. Figure 6 considers the throughput performance under different transmit power at (S). It is clear that the highest throughput can be obtained regardless of the increase in the transmit power at (S), and the tight upper bound is close to the simulation results. The throughput performance changes considerably, if we choose different values of the SNR threshold. As a result, the relay with EH circuitry can satisfy proper applications for wireless communications with an acceptable SNR level.

Similarly, we consider the throughput performance under the impact of self-interference in Fig. 7. It is clear that the throughput declines as self-interference increases compared to SP's upper bound which is equal to 0 . The performance is limited by both SNR threshold, at $0 \mathrm{~dB}$ and $2 \mathrm{~dB}$, respectively.

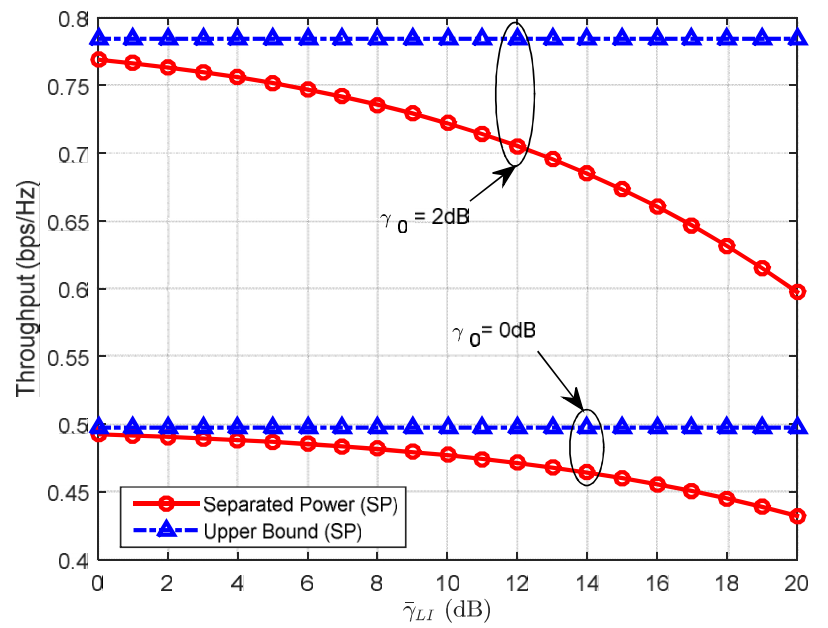

Fig. 7. The asymptotic throughput performance under impact of selfinterference with $\bar{\gamma}_{S R}=\bar{\gamma}_{R D}=20 \mathrm{~dB}$.

Finally, Fig. 8 illustrates the transmission rate in terms of the optimal time switching coefficients. In case of SP, since it cannot harvest energy to assist the communication process, the transmission rate remains unchanged. Thanks to EH capacity and the installation of battery store at (R), HP outperforms SP in terms of higher transmit power.

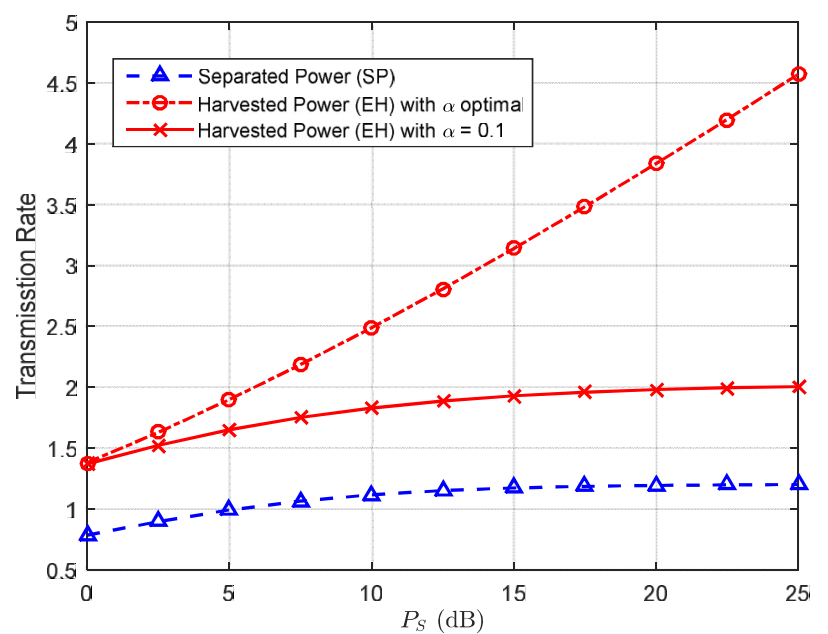

Fig. 8. The transmission rate vs $P_{S}$ other parameters: $\bar{\gamma}_{L I}=5 d B, \bar{\gamma}_{S R}=\bar{\gamma}_{R D}=20 d B, \alpha=0.1$ and $\alpha_{o p t}$. 


\section{CONCLUSIONS}

In this paper, we propose wireless power supply policies in FD relaying networks. In particular, in HP mode, the choice of optimal time with the help of the EH protocol assist the relay in sharing energy without consuming totally it like SP. The exact expressions for outage probability and throughput are provided.

Numerical results confirm that the simulation results match well with the exact expression for outage probability. Besides that, the numerical results help gain an acceptable outage performance between HP and SP mode. We give some valuable insights into the impact of self-interference, time switching coefficients and the transmit power on the system throughput.

\section{APPENDIX A}

\section{Proof of Proposition 1}

This appendix derives the outage probability at (D) for TSR protocol. We obtain the PDF and CDF of SNR in the first hop as below.

The PDF is given by

$$
f_{|h|^{2}}(x)=\frac{1}{\Omega_{h}} \exp \left(-\frac{x}{\Omega_{h}}\right),
$$

where $x \geq 0$. The CDF can be computed as

$$
F_{|h|^{2}}(x)=1-\exp \left(-\frac{x}{\Omega_{h}}\right) \text {. }
$$

We have the distribution function of self-interference as below

$$
f_{|f|^{2}}(x)=\frac{1}{\Omega_{f}} \exp \left(-\frac{x}{\Omega_{f}}\right) \text {. }
$$

Next, we calculate the outage probability of the overall system as

$$
\begin{aligned}
F_{\gamma_{R}}(x)= & \operatorname{Pr}\left\{\gamma_{R}<x\right\}=\operatorname{Pr}\left\{\frac{P_{S}|h|^{2}}{P_{R}|f|^{2}}<x\right\}= \\
& =1-\frac{P_{S} \Omega_{h}}{P_{R} \Omega_{f} x+P_{S} \Omega_{h}} .
\end{aligned}
$$

It is noted that end-to-end SNR in DF scheme can be calculated as $Y=\min \left\{\gamma_{R}, \gamma_{D}\right\}$. We have the general outage probability

$$
\begin{gathered}
F_{Y}(x)=1-\left(1-F_{\gamma_{D}}(x)\right) \times\left(1-F_{\gamma_{R}}(x)\right)= \\
=1-P_{S} \Omega_{h}\left(P_{S} \Omega_{h}+P_{R} \Omega_{f} x\right)^{-1} \exp \left(-\frac{\sigma^{2}}{P_{R} \Omega_{g}} x\right)
\end{gathered}
$$

where $F_{\gamma_{D}}(x)=1-\exp \left\{-\frac{\sigma^{2}}{P_{R} \Omega_{g}} x\right\}$.
This ends the proof for Proposition 1.

\section{APPENDIX B}

Proof of Proposition 2

Similarly, we first have PDF of the SNR for selfinterference

$$
f_{|f|^{2}}(x)=\frac{1}{\Omega_{f}} \exp \left(-\frac{x}{\Omega_{f}}\right)
$$

The CDF of the SNR at relay node as

$$
F_{\gamma_{R}}(x)=1-\left(1-\exp \left(-\frac{1}{\rho x \Omega_{f}}\right)\right) \text {. }
$$

Using the integral identity [16], the outage probability can be calculated as

$$
F_{\gamma_{D}}(x)=1-2 \sigma^{2} \sqrt{\frac{x}{\rho P_{S} \Omega_{h} \Omega_{g}}} K_{1}\left(2 \sigma^{2} \sqrt{\frac{x}{\rho P_{S} \Omega_{h} \Omega_{g}}}\right) .
$$

Finally, we compute the overall outage probability as

$$
\begin{gathered}
P_{\text {out }}^{H P}(x)=1-2 \sigma^{2}\left(1-\exp \left(-\frac{1}{\rho x \Omega_{f}}\right)\right) \times \\
\times \sqrt{\frac{x}{\rho P_{S} \Omega_{h} \Omega_{g}}} K_{1}\left(2 \sigma^{2} \sqrt{\frac{x}{\rho P_{S} \Omega_{h} \Omega_{g}}}\right) .
\end{gathered}
$$

This ends the proof for Proposition 2.

\section{REFERENCES}

[1] K. Yamamoto, K. Haneda, H. Murata, S. Yoshida, "Optimal transmission scheduling for hybrid of full-duplex and half-duplex relaying", IEEE Commun. Lett., vol. 15, no. 3, pp. 305-307, 2011. [Online]. Available: http://dx.doi.org/10.1109/LCOMM.2011. 011811.101925 .

[2] S. Bi, C. K. Ho, R. Zhang, "Wireless powered communication: op portunities and challenges", IEEE Commun. Mag., vol. 53, no. 4, pp. 117-125, 2015. [Online]. Available: http://dx.doi.org/10.1109 /MCOM.2015.7081084.17

[3] X. Lu, P. Wang, D. Niyato, D. I. Kim, Z. Han, "Wireless networks with RF energy harvesting: a contemporary survey", IEEE Communications Surveys \& Tutorials, vol. 17, no. 2, pp. 757-789 2015. [Online]. Available: http://dx.doi.org/10.1109/COMST.2014. 2368999.

[4] I. Krikidis, S. Timotheou, S. Sasaki, "RF energy transfer for cooperative networks: Data relaying or energy harvesting?", IEEE Commun. Lett., vol. 16, no. 11, pp. 1772-1775, 2012. [Online]. Available: http://dx.doi.org/10.1109/LCOMM.2012.091712.121395

[5] H. Ju, R. Zhang, "User cooperation in wireless powered communication networks", in Proc. IEEE GLOBECOM, 2014, pp. 1430-1435. [Online]. Available: http://dx.doi.org/10.1109/ GLOCOM.2014.7037009

[6] A. A. Nasir, X. Zhou, S. Durrani, R. A. Kennedy, "Relaying protocols for wireless energy harvesting and information processing", IEEE Trans. Wireless Commun., vol. 12, no. 7, pp. 622-3636, 2013. [Online]. Available: http://dx.doi.org/10.1109/TWC.2013.062413. 122042

[7] Z. Ding, I. Krikidis, B. Sharif, H. V. Poor, "Wireless information and power transfer in cooperative networks with spatially random relays", IEEE Trans. on Wireless Commun., vol. 13, no. 8, pp. 4440-4453, 2014. [Online]. Available: http://dx.doi.org/10.1109/TWC.2014. 2314114

[8] H. Ju, R. Zhang, "Optimal resource allocation in full-duplex wireless 
powered communication network", IEEE Trans. on Commun., vol. 62, no. 10, pp. 3528-3540, 2014. [Online]. Available: http://dx.doi.org/10.1109/TCOMM.2014.2359878.

[9] C. Zhong, H. A. Suraweera, G. Zheng, I. Krikidis, Z. Zhang, "Wireless information and power transfer with full-duplex relaying", IEEE Trans. Commun., vol. 62, no. 10, pp. 3447-3461, 2014 [Online]. Available: http://dx.doi.org/10.1109/TCOMM.2014. 2357423

[10] V. Aggarwal, M. Duarte, A. Sabharwal, N. K. Shankaranarayanan, "Full-duplex or half-duplex? a capacity analysis with bounded radio resources", IEEE Information Theory Workshop, 2012, pp. 207-211. [Online]. Available: http://dx.doi.org/10.1109/ITW.2012.6404659

[11] P. Fazio, F. De Rango, C. Sottile, "A new interference aware on demand routing protocol for vehicular networks", in Proc. of Int. Symposium on Performance Evaluation of Computer \& Telecommunication Systems (SPECTS), 2011, pp. 98-10.

[12] P Fazio, F De Rango, C Sottile, C Calafate, "A new channel assignment scheme for interference-aware routing in vehicular networks", in Proc. 3rd IEEE Vehicular Technology Conf. (VTC
Spring), 2011, pp. 1-5. [Online]. Available: http://dx.doi.org/ 10.1109/VETECS.2011.5956777

[13] F. Suriano, F. De Rango, P. Popovski, "Opportunistic Interference Cancellation evaluation in cognitive radios under power control strategies", in Proc. 9th Int. Wireless Communications and Mobile Computing Conf. (IWCMC), 2013, pp. 609-614. [Online]. Available: http://dx.doi.org/10.1109/IWCMC.2013.6583627

[14] F. De Rango, A. Panzarella, S. Marano, "Controlled Interference Mitigation MAC for UWB networks with quality of service support", in Proc. IEEE Int. Conf. Mobile Adhoc and Sensor Systems, 2007, pp. 1-3. [Online]. Available: http://dx.doi.org/10.1109/MOBHOC. 2007.4428678

[15] C. Zhong, H. A. Suraweera, G. Zheng, I. Krikidis, Z. Zhang, "Wireless information and power transfer with full duplex relaying", IEEE Trans. Communications, vol. 62, no. 10, pp. 3447-3461, 2014 [Online]. Available: http://dx.doi.org/10.1109/TCOMM.2014 2357423

[16] I. S. Gradshteyn, I. M. Ryzhik, Table of Integrals, Series, and Products. Academic Press, 5th ed, 1994. 